\title{
El nuevo enfoque en los ensayos mecánicos de la madera aserrada para uso estructural en la normativa europea
}

\author{
Iñigo Ariza López ${ }^{1}$
}

\begin{abstract}
RESUMEN
La madera es quizá uno de los materiales dentro de la construcción que posee una regulación a nivel de normas y ensayos más precaria e insuficiente desde el punto de vista práctico o del profesional. Así lo asumen con frecuencia los profesionales de países meridionales. En este contexto, la aparición de un cuerpo de normativa de usos y ensayos, a nivel de laboratorio, común para toda la Comunidad Económica Europea no sólo debe interesar a los directamente afectados, como pueden ser profesionales europeos, sino también a todos aquellos productores que quieran introducir sus productos en Europa, ya que esto no será posible sin efectuar dichos ensayos. Además, se suma el interés propio de las novedades de esta normativa, en lo que a ensayos se refiere. Estos ensayos poseen un concepto muy distinto del material madera de como se ha hecho hasta ahora en prácticamente toda la normativa a nivel mundial. A dicha forma de trabajar se le llama "nuevo enfoque" y trae como consecuencias una mayor seguridad en el uso de la madera y mayores requerimientos en las instalaciones de los laboratorios de ensayos. En este documento se procede a señalar estas cuestiones y a hacer una ligera comparación (sin pretender ser exhaustivos) que haga comprender a grandes rasgos, los puntos esenciales de este "nuevo enfoque".
\end{abstract}

PALABRAS CLAVE:

Comunidad Europea, construcción, ensayos, madera, normativa, nuevo enfoque.

\begin{abstract}
Timber is perhaps one of the building materials with a meager and insufficient regulations (standards and testing methods) from the practical an professional point of view, and it is considered so by the professional from Mediterranean countries. In this context the publication of a set of standards for uses and testing, for laboratory purposes, which is common for the entire European Community should not only be of interest to those directly involved but for those producers aiming to commercialize their products in Europe, which will not be possible without making that type of tests. Testing set of this kind implies a very different concept of wood a a structural material unlike anything done elsewhere. This new methodology I called a "new approach" and it is aimed at increasing safety in the use of timber and greater requirement in the testing facilities. This paper detail the main points of this "new approach" without trying to be exhaustive but intending highlight those points.
\end{abstract}

KEY WORDS:

European Community, construction, essays, wood, technical regulation, new approach. 


\section{INTRODUCCIÓN}

Europa es una consumidora importante de madera a nivel mundial. De forma aproximada, se puede decir que el Viejo Continente importa un total de 33,5 millones de metros cúbicos frente a los 22 millones que suponen el mercado en su interior (y de este mercado interior el $90 \%$ procede de los países escandinavos, lo que da idea de su déficit). La producción maderera del cono sur de América resulta ser poco importante en este total de la madera que consume Europa. Este destino, sin embargo, supone una fracción importante de las exportaciones que hacen los países sudamericanos.

En este contexto de intercambio de materias primas a nivel mundial, nos encontramos con una gran diversidad en la normativa técnica referente a la madera, lo que resulta poco conveniente para el mercado. Sin perjuicio de lo dicho, el espíritu de estas normativas técnicas en los distintos países suele ser muy similar. Es usual que las normas de algunos países tengan un referente claro en las normas más antiguas de países con experiencia reconocida en dicho campo. En este contexto podemos citar como ejemplos las normas de los EEUU, ANSI; o las normas COPANT en Sudamérica, que son un interesante ejemplo de unificación en esta materia de varios países. En Europa existía una atomización completa de la normativa, país por país.

\section{LA NUEVA NORMATIVA DE ENSAYOS EUROPEA}

La nueva normativa de ensayos europea que se presenta posee un interés y una novedad doble. Por un lado, todo entendido estará atento a los cambios que se producen a su alrededor, más cuando se trata de una normativa que unificará la de todos los países de la Unión Europea, con consecuencias relevantes para las importaciones. Ello surge de la directiva comunitaria 89/106/CEE, que obliga a la unificación normativa dentro de la Comunidad. Por ello, todas las normativas nacionales se hallan en proceso de sustitución. En el caso de la madera para uso estructural, los cambios son importantes y afectan a la denominación, a la forma de obtención de los valores mecánicos, a la clasificación del producto de cara al consumo, etc. Por tanto tiene un interés evidente para los exportadores, añadido a la simplificación que supone tratar con una normativa única.

Además, en el caso de la madera, son frecuentes las exportaciones desde países productores: Canadá, Estados Unidos, Chile, Rusia y países del Norte de Europa. Así, es usual que la normalización o los distintivos de calidad (de forma genérica) se basen en el prestigio del país productor o incluso en el sistema propio de cada aserradero.

Las normas existentes en la Europa comunitaria hasta ahora eran bastante parecidas entre sí en lo referente a los ensayos, sobre todo en la forma de entender la madera como material resistente. Pertenecían al llamado "enfoque de las tensiones básicas". Según esta forma de trabajar, para obtener los esfuerzos mecánicos se ensayaban probetas de tamaño pequeño y en general libres de defectos, ya que estos tamaños permiten la exclusión de defectos relevantes y normales en piezas, como pueden ser nudos, fendas o incluso una desviación generalizada de la fibra. Dentro de este enfoque era normal rechazar probetas con ciertos defectos. En la figura 1 se representa la forma de obtener probetas según las normas UNE españolas en función del diámetro de la troza; en ella se señala la zona útil. Como se puede observar, no se utiliza la médula o zona central del tronco. 


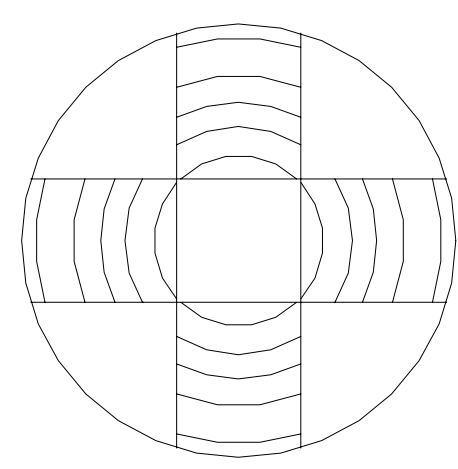

Diámetro $\leq 180 \mathrm{~mm}$

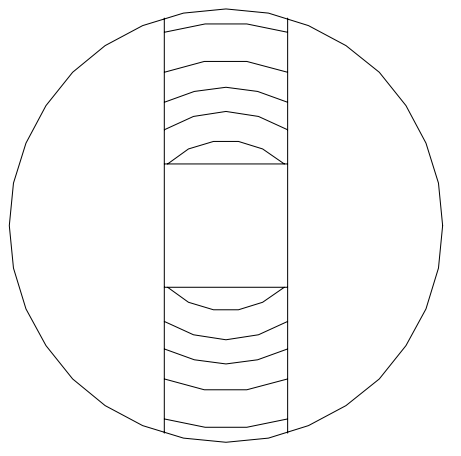

Diámetro > $180 \mathrm{~mm}$

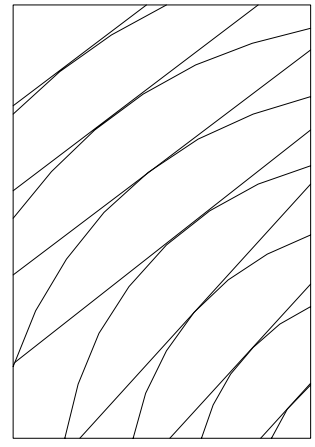

Figura 1. Obtención de la probeta. Tomado de la norma UNE 56-528.

Otras normas, como las COPANT en América, aplicaban un enfoque similar. En este caso, las dimensiones de las probetas eran algo mayores. En cualquier caso, eran piezas con dimensiones que pueden oscilar entre los $20 \mathrm{~mm}$ y los $50 \mathrm{~mm}$ de lado por $70 \mathrm{~mm}$ a $750 \mathrm{~mm}$ de longitud, según la norma y el tipo de ensayo.

El origen de esta forma de entender los ensayos puede ser variado. En un enfoque estadístico simple, se pretende realizar ensayos sobre probetas de un tamaño que sería representativo en otros materiales con mayor homogeneidad en su constitución, como el hormigón o el acero. La madera es quizá, de entre los que se usan en la construcción, el material que posee mayores diferencias en su comportamiento. Se trata del tejido de un ser vivo, que se ha ido adaptado a situaciones muy diversas; hay incluso diferencias importantes dentro de un mismo individuo. Casi no hay mejora genética, existiendo gran diversidad y entrecruzamiento. Como consecuencia, entre especies afines es muy posible que se obtengan más diferencias entre los distintos individuos que las debidas a cualquier otro parámetro.
Precisamente, la dificultad de obtener una pieza para probeta con las características perfectamente homogéneas ha llevado a tamaños tan reducidos de probetas con una calidad muy alta. También es posible que ello provenga de una época en la que se podía disponer de piezas muy buenas de madera aserrada, con pocos defectos. Con estos ensayos se obtenían las llamadas tensiones básicas para cada especie. No se obtendrían tensiones características como en el resto de los materiales; la falta de confianza llevaba al concepto de las tensiones básicas, estableciendo una diferencia entre la madera y cualquier otro material de construcción. Al obtenerse dichas tensiones básicas, distintas de las tensiones de cálculo, se tienen que aplicar minoraciones mediante una serie numerosa de tablas de uso frecuente, en las que se listaban los defectos más comunes o bien cada cual utilizaba su experiencia.

Por tanto, en esta forma de tratar el material existían dos aspectos llamativos: un manejo estadístico pobre e inadecuado, si se tiene en cuenta la naturaleza heterogénea de la madera, muy distinta a la de 
otros materiales y el uso poco claro de esta "traducción" de los resultados de los ensayos a la realidad del uso de la madera.

El segundo punto de interés de la nueva normativa radica en el enfoque novedoso que se emplea para la madera de uso estructural, sobre el que habrá una explicación más amplia más adelante. Se trata de ensayos sobre piezas de madera aserrada con las dimensiones comerciales de cada procedencia o aserradero, es decir, en general, vigas o tablones. Se tienen en cuenta, por tanto, las posibilidades de aserrado que permite la madera. Se ensaya una cantidad importante de piezas en número y volumen total, con los defectos comunes de éstas provocados por el aserrado y secado, una vez clasificadas en calidades estructurales; incorporando a los resultados mecánicos, la incertidumbre que supone la inclusión de los defectos normales en elementos de gran tamaño, tal y como salen del aserrado. Se obtendrán los valores de resistencias características y no de tensiones básicas como ocurría antes, para así unificar su utilización con la de cualquier otro material resistente $y$, lo que es más importante, un mayor nivel de confianza en estos ensayos. Se debe tener presente que se entiende por valores característicos aquellos que tienen una probabilidad del $95 \%$ de ser superados, para el cálculo los valores característicos se ponderan (reducen) con los coeficientes para cada material.

\section{LA NUEVA NORMATIVA EN SU CONJUNTO}

Antes de entrar de lleno en los ensayos se debe entender el ámbito en el que se inscriben éstos y que resulta en la práctica tan importante o más que los propios ensayos. Estos ensayos se aplican para determinar las resistencias características, que tendrán carácter normativo a partir de entonces. Esto se realiza, dado el volumen importante de material y dispositivos, por laboratorios con cierta entidad y capacidad. Los nuevos ensayos no son adecuados para el control de calidad, aunque sí pueden ser un precedente normalizado de ensayos de elementos resistentes enteros antes de su colocación en obra o incluso apeados, como sería un caso posible en las rehabilitaciones.

Para las bases teóricas y prácticas del cálculo de la madera en estructuras se establece el Eurocódigo Estructural n 5 , que posee adaptaciones en cada país. En esta normativa se designa el material resistente madera con una letra (" $C$ " en madera aserrada para conífera y "D" para frondosa o angiosperma), seguido de la resistencia característica a flexotracción expresada en $\mathrm{MPa}\left(\mathrm{N} / \mathrm{mm}^{2}\right)$. Existen valores desde C-14 hasta C-28, en coníferas, por ejemplo, por lo que el cálculo y la denominación es similar al de otros materiales estructurales.

Para llegar en la práctica a obtener dichas resistencias características es necesaria la calidad estructural del material, obtenida ya sea por la llamada clasificación visual o con la clasificación mecánica. La clasificación visual utiliza los defectos aparentes de la madera tales como fibra, anillos, nudos, etc., siendo similar a la de otros países. Para dicha clasificación se usan los mismos criterios en cada país miembro, sólo que varía el ámbito de las especies a las que se aplica. Actualmente están aún en proceso de revisión las UNE-EN 518 y UNE-EN 519, que en España son las UNE 56-544 para clasificación visual de madera aserrada de conífera de uso estructural y UNE 56-545 para clasificación mecánica de madera aserrada, respectivamente. Las calidades estructurales obtenidas son $1^{\underline{a}}, 2^{\underline{a}} y$, según los países, una 3", más el "rechazo".

Posteriormente, necesitaremos saber relacionar estas calidades con valores de 
resistencia característica aceptada. El conjunto de normas implicadas son las que se presentan en la Tabla 1.

En la norma EN 338 se establecen los conceptos generales sobre clases resistentes y la obtención de las resistencias características. Las condiciones de muestreo e interpretación de los ensayos se dan en la norma EN 384, en la cual se establece además la posibilidad de comparar estos ensayos con otros sobre probetas "pequeñas libres de defectos", tal como las cita textualmente. Los ensayos de propiedades mecánicas paralelas a las fibras y densidad se definen en la norma EN 408 y los ensayos de propiedades perpendiculares a la fibra y esfuerzos de corte o desgarro se ven en la norma EN
1193. En todo este proceso se busca una correlación entre las clases de madera aserrada estructural y las propiedades en valores característicos, ya sean mecánicas o físicas (caso de la densidad). Esta correlación se acepta a partir de entonces para cada grupo de especie + procedencia + calidad, lo que da una resistencia característica a flexotracción y con ella el resto de propiedades necesarias para el cálculo. Dichas correlaciones se publican también en forma de norma, en este caso la EN 1912. Por tanto, éste es proba blemente el apartado más importante de cara a la comercialización y construcción. Se puede observar que en la relación de especie-resistencia se incluyen maderas foráneas (Tabla 2).

Tabla 1. Nueva normativa europea sobre los estados límite

\begin{tabular}{ll}
\hline UNE-EN 844 & Madera aserrada y madera en rollo. Terminología. \\
\hline UNE-EN 338 & Madera estructural. Clases resistentes. \\
\hline UNE-EN 384 & $\begin{array}{l}\text { Madera estructural. Determinación de los valores característicos de } \\
\text { las propiedades físicas y mecánicas. }\end{array}$ \\
\hline UNE-EN 336 & Madera estructural. Coníferas y chopo. Dimensiones y tolerancias. \\
\hline UNE-EN 408 & $\begin{array}{l}\text { Madera aserrada y madera laminada encolada para uso estructural. } \\
\text { Determinación de algunas propiedades físicas y mecánicas. }\end{array}$ \\
\hline UNE-EN 1193 & $\begin{array}{l}\text { Estructuras de madera. Madera estructural y madera laminada } \\
\text { encolada. Determinación de la resistencia al esfuerzo cortante y de } \\
\text { las propiedades mecánicas en dirección perpendicular a la fibra }\end{array}$ \\
\hline UNE-EN 518 & $\begin{array}{l}\text { Madera estructural. Clasificación. Requisitos de las normas de } \\
\text { clasificación visual resistente. }\end{array}$ \\
\hline UNE 56-544:99 & $\begin{array}{l}\text { Clasificación visual de la madera aserrada para uso estructural: } \\
\text { madera de coníferas. }\end{array}$ \\
\hline UNE-EN 519 & $\begin{array}{l}\text { Madera estructural. Clasificación. Requisitos para la madera } \\
\text { clasificada mecánicamente y para las máquinas de clasificación. }\end{array}$ \\
\hline UNE-EN 56-545 & Marcado de la madera clasificada. \\
\hline EN 1912 & Asignación de especies y calidades visuales \\
\hline
\end{tabular}


Tabla 2. Relación de especie-resistencia. Tomado de la norma europea EN 1912:98

\begin{tabular}{|c|c|c|c|c|}
\hline $\begin{array}{l}\text { CLASE } \\
\text { RESISTENTE }\end{array}$ & $\begin{array}{c}\text { PAÍS QUE } \\
\text { PUBLICA LA } \\
\text { NORMA DE } \\
\text { CLASIFICACIÓN* }\end{array}$ & CALIDAD* & $\begin{array}{c}\text { NOMBRE } \\
\text { COMERCIAL }\end{array}$ & PROCEDENCIA \\
\hline D70 & Reino Unido & $\begin{array}{l}\mathrm{HS} \\
\mathrm{HS}\end{array}$ & $\begin{array}{c}\text { Balau } \\
\text { Greenheart }\end{array}$ & $\begin{array}{c}\text { Sudeste de Asia } \\
\text { Guayana }\end{array}$ \\
\hline D60 & $\begin{array}{l}\text { Países Bajos } \\
\text { Reino Unido }\end{array}$ & $\begin{array}{l}\text { A/B } \\
\mathrm{HS} \\
\mathrm{HS} \\
\mathrm{HS}\end{array}$ & $\begin{array}{l}\text { Azobé } \\
\text { Ekki } \\
\text { Kapur } \\
\text { Kempas }\end{array}$ & $\begin{array}{l}\text { Oeste de África } \\
\text { Oeste de África } \\
\text { Sudeste de Asia } \\
\text { Sudeste de Asia }\end{array}$ \\
\hline D50 & Reino Unido & $\begin{array}{l}\mathrm{HS} \\
\mathrm{HS} \\
\mathrm{HS} \\
\mathrm{HS}\end{array}$ & $\begin{array}{l}\text { Keruing } \\
\text { Cari } \\
\text { Opepe } \\
\text { Merbau }\end{array}$ & $\begin{array}{c}\text { Sudeste de Asia } \\
\text { Oeste de Australia } \\
\text { Este de África } \\
\text { Sudeste de Asia }\end{array}$ \\
\hline D40 & Reino Unido & $\begin{array}{l}\mathrm{HS} \\
\mathrm{HS} \\
\mathrm{HS}\end{array}$ & $\begin{array}{c}\text { Iroko } \\
\text { Jarrah } \\
\text { Teca }\end{array}$ & $\begin{array}{c}\text { África } \\
\text { Este de Australia } \\
\text { Sudeste de Asia }\end{array}$ \\
\hline D35 & - & & & \\
\hline D30 & - & & & \\
\hline
\end{tabular}

Entre estas normas se señala el valor práctico que posee la clasificación visual que, al ser bastante sencilla (reducida a dos o tres clases de resistencia y unificada) e ilustrativa acerca de los defectos de la madera, se puede usar incluso en la recepción del material en obra.

Con todo ello se puede decir que la nueva normativa de ensayo, sin perder su novedad, es una parte en el conjunto de normativas europeas sobre madera.

\section{EL NUEVO MÉTODO DE ENSAYOS EN MADERA ASERRADA PARA USO ESTRUCTURAL}

Este apartado se referirá a algunos aspectos de las normas EN-384 y 408. Como se mencionó antes, en el llamado nuevo enfoque se ensayan las propiedades mecánicas en elementos de tamaño comercial, según las "dimensiones representativas de cada procedencia o aserradero" (EN 384). No existen probetas en el sentido tradicional de pieza extraída o seleccionada, se ensayan lo que pueden ser elementos estructurales completos con sus defectos, tal y como se obtienen del aserrado y dimensionado de sus caras.

A primera vista, resalta además del tamaño de las probetas (aspecto que se verá más adelante), el tamaño de las muestras, pues se aplica un enfoque estadístico que usa una gran cantidad de material. Cada muestra consiste de un grupo de probetas con dimensiones iguales, de forma que se seleccionan los tamaños más representativos (escuadría y longitud) que se pueden obtener para una 
especie y procedencia determinados. Las muestras poseen como mínimo 40 probetas. En la figura 2 se ilustra el coeficiente $k$ que penaliza el valor final de la resistencia característica en la madera cuando $k<1$. Para no sufrir estas penalizaciones se necesitarían al menos 200 probetas en total, repartidas en 5 muestras distintas, o bien 400 probetas en total repartidas en sólo 4 muestras con 100 probetas cada una. Es interesante comparar estas cantidades totales de probetas con las de las normas de ensayos anteriores, 200 frente a 50 ó 60 de las normas UNE (normas españolas) o alrededor de 30 en las normas COPANT, ambas para ensayos de flexión, y todo ello referido a valores comunes en la dispersión de los resultados (Fig. 2). Este hecho, unido a una serie de ensayos sobre las probetas bastante más exhaustivos que los de cualquier norma hasta el momento, hace necesarias unas instalaciones y personal abundante.
Se deben ensayar las dimensiones (secciones) usuales del aserrado para uso estructural de cada procedencia, que como es sabido, está limitado por el tamaño y calidad de las trozas. El canto de referencia es de $150 \mathrm{~mm}$; la longitud para los ensayos de flexión y cortante, es de al menos 19 veces la mayor dimensión transversal. Para los ensayos de tracción y compresión es necesaria una longitud de sólo 18 y 9 veces el canto, respectivamente. En la práctica, salvo el caso de grandes despieces o árboles con mucha desviación en la fibra, se limitará más la sección máxima que se obtiene de cada procedencia y no la longitud de la viga. Comparando esas dimensiones (para un canto de $150 \mathrm{~mm}$, se tendría una longitud de al menos 2,85 m) con las dimensiones de $50 \mathrm{~mm} \times 50 \mathrm{~mm} \times 750 \mathrm{~mm}$, en las normas COPANT o incluso los $30 \mathrm{~mm} x$ $30 \mathrm{~mm} \times 300 \mathrm{~mm}$, de las normas españolas, para ensayos de flexión.

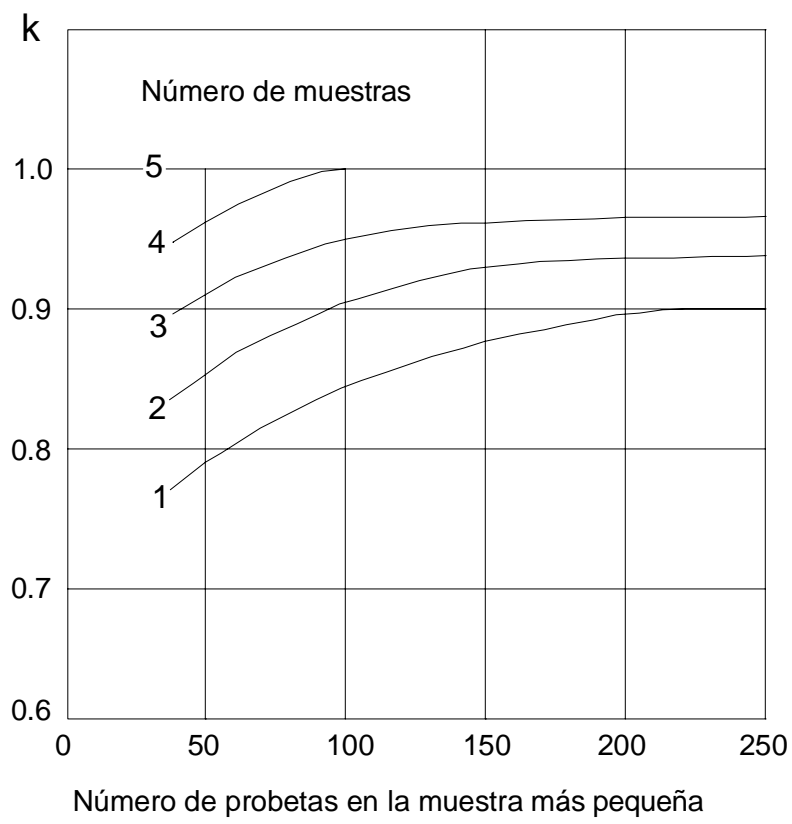

Figura 2. Tamaño de muestra mínimo y penalización de la resistencia característica, dependiendo del número de probetas.

Tomado de la norma UNE-EN-384. 
En España estos ensayos se han realizado hasta el momento con especies de coníferas (Pinus sylvestris L., Pinus nigra Arn., Pinus pinaster Ait., Pinus radiata $D$. Don.), por los laboratorios del INIA en Madrid y Valsaín.

Previamente a los ensayos mecánicos se acondicionan las probetas a un $65 \%$ de humedad relativa y $20{ }^{\circ} \mathrm{C}$ de temperatura, en estas condiciones ambientales las resinosas se estabilizan alrededor de un $12 \%$ de contenido de humedad aproximadamente. Se determinan las dimensiones, la densidad y el contenido en agua. Tanto el peso específico como el contenido de humedad se volverán a comprobar en todas la probetas una vez rotas las piezas. El contenido de humedad obtenido por desecación servirá, además, para relacionarlo con los datos registrados con higrómetro de resistencia, que son mucho más fáciles de obtener, pero aproximados.

Todas las probetas se marcan según la clasificación visual o mecánica o incluso relacionando ambas clasificaciones de forma simultánea en cada viga. En cualquier caso, además del marcado normativo y otras referencias de la probeta se señalan los defectos que aparecen en la llamada zona crítica, que para estos ensayos con dos puntos de aplicación de la carga corresponde al tercio central de la viga. Así, la clasificación obtenida y los defectos observados se relacionan con los resultados experimentales posteriomente.

La determinación de los valores relacionados con la flexión comprende, a diferencia de enfoques anteriores, no uno, sino una serie de ensayos, que culminan con la rotura de la probeta (ensayo de resistencia). Se empieza por la determinación de los módulos de elasticidad. Debido a la dificultad de deslindar la influencia en la deformación de una viga de madera de tamaño real de los componentes de la flexión, del cortante e incluso de fenómenos menos teóricos pero mucho más relevantes en una medida precisa, como es el aplastamiento en los apoyos y en los puntos de aplicación de las cargas, los ensayos poseen dispositivos más complejos que los vistos hasta ahora y formulaciones que no resultan inmediatas.

En primer lugar se describirá el ensayo de determinación del módulo de elasticidad en flexión, aunque no se trate del primero que necesariamente se realiza, sí es el más ilustrativo y quizá el más característico de todos ellos.

Las condiciones generales del ensayo son similares a otros: la humedad estará estabilizada en torno al 12\%; la velocidad de carga $(\mathrm{mm} / \mathrm{s})$ debe ser constante de tal manera que las deformaciones sean menores de 0,003 h; y la exactitud en la medida será del $1 \%$ ó $0,02 \mathrm{~mm}$ para flechas inferiores a $2 \mathrm{~mm}$. Se permite el uso de placas de reparto y topes laterales para evitar el vuelco o pandeo lateral.

El dispositivo de ensayo general (Fig. 3) tiene una separación mínima entre apoyos de 18 veces la mayor dimensión transversal y la longitud de la pieza deberá ser al menos, 19 veces esta medida. La carga se aplica en dos puntos, separados entre sí 6 veces el canto. Las mejoras con respecto a los ensayos tradicionales son: el diagrama de momentos flexionantes no es triangular (al igual que en las normas americanas), lo que se acerca más a los estados usuales de carga. Además, esta disposición permite ensayar con esfuerzos de flexión constantes el tercio central de la viga, llamada por la norma "zona crítica", por lo que en este tercio central la viga se romperá por la zona más débil. Esto permite hacer una correlación con los defectos visibles o con las marcas obtenidas de la clasificación mecánica. Todo ello se recogerá en el llamado informe de ensayo, suponiendo que en un 


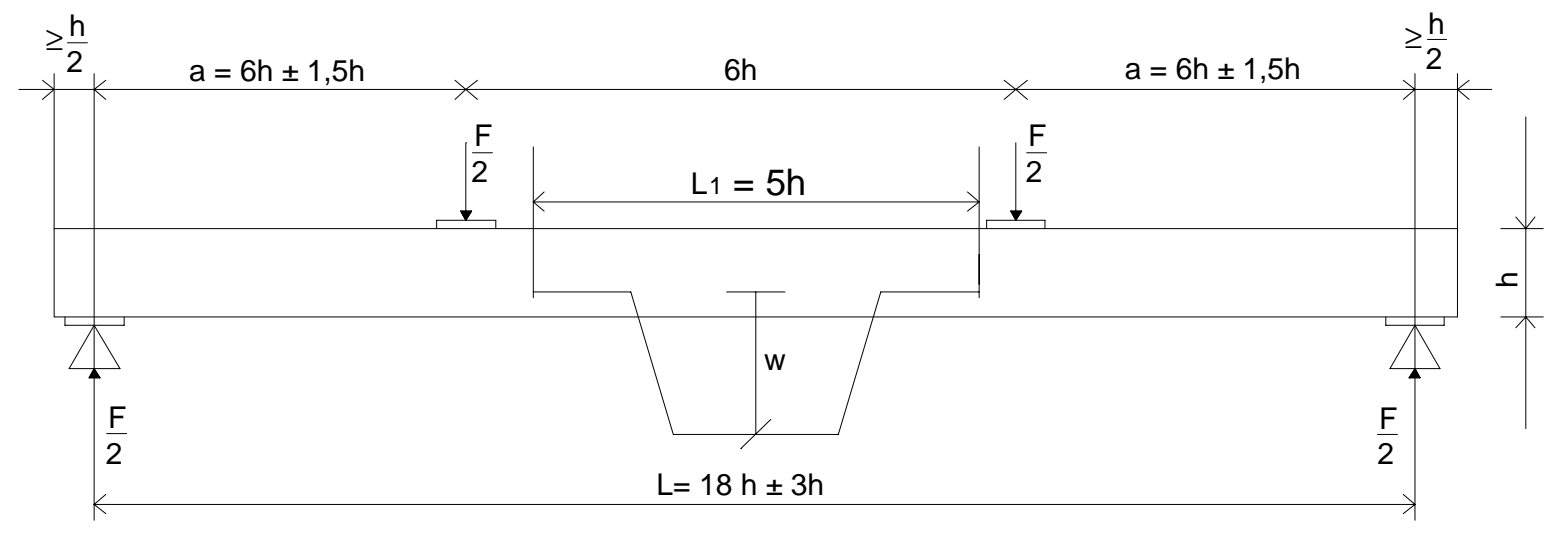

Figura 3. Dispositivo de módulo en flexión. Tomado de UNE-EN 408.

estado de carga triangular, la viga tenderá a romperse en el centro, aunque quizá posea defectos determinantes en otras partes, de forma que la influencia de estos será menos evidente, y ello resulta de interés importante en un material tan heterogéneo como la madera.

En los ensayos de determinación del módulo de elasticidad no se debe llegar a rebasar el límite elástico ni a dañar permanentemente la probeta, para que esté disponible para las siguientes pruebas.

El ensayar probetas de este tamaño permite además tener un control mayor mediante dispositivos más complejos como el de la figura. Por ejemplo, se evita la incidencia del aplastamiento en los apoyos mediante el uso de la llamada percha, pieza en V (Fig. 4), con la que se miden las deformaciones en la parte central ( 5 veces el canto de la pieza), sin llegar ni siquiera a los puntos de aplicación de carga. Además con esta percha se recogen las deformaciones sólo en el tercio central, en una zona con momento flexionante constante (Ensayo del módulo en flexión, Lab. INIA, Madrid). Por tanto la obtención del módulo es:

$$
E_{m}=\frac{a \cdot l_{1}^{2}\left(F_{2}-F_{1}\right)}{16 \cdot l\left(w_{2}-w_{1}\right)}
$$

donde:

$F_{1}=$ carga inicial

$F_{2}=$ carga final

$w_{1}=$ deformación inicial

$w_{2}=$ deformación final

$E_{m}=$ módulo de elasticidad en flexión

$a$ = distancia desde el punto de aplicación de la carga hasta el apoyo más cercano

$I_{1}=$ distancia entre apoyos de la percha

$I$ = momento de inercia de la sección

Los ensayos de determinación del módulo de cortante se pueden realizar de dos maneras, por el método del claro único, más sencillo, o por el del claro variable, compuesto por una serie de 4 ensayos sobre la misma viga. El módulo de cortante es un valor difícil de obtener experimentalmente pero muy útil para efectos de cálculo.

La determinación del módulo de cortante por el método del claro único es más fácil de obtener experimentalmente, aunque más inexacto. Se obtienen dos módulos, el módulo de elasticidad aparente y el módulo de cortante (Fig. 4). También 
se utiliza la percha con objeto de reducir la influencia del aplastamiento cercano a los apoyos, ya que ésta se coloca alejada de la superficie de apoyo. Para obtener el módulo de cortante es necesario haber calculado anteriormente el módulo de elasticidad en flexión.

En el método del claro variable, el dispositivo es similar, pero se repite la operación al menos cuatro veces en cada viga. Parece ser más preciso, aunque los dos sean igualmente válidos. Se usa una combinación de diferencias $(\mathrm{h} / \mathrm{l})^{2}$ aproximadamente iguales; para el canto de referencia de $150 \mathrm{~mm}$, resultaría una serie de aproximadamente $3000 \mathrm{~mm}, 1300 \mathrm{~mm}$, $965 \mathrm{~mm}$ y $800 \mathrm{~mm}$ de claro, respectivamente. La obtención del resultado numérico no es inmediata. Se calculan los módulos de elasticidad aparentes para cada claro, se hace una gráfica contra los valores $(\mathrm{h} / \mathrm{l})^{2}$ (Fig. 5) encontrándose una regresión lineal (pendiente $\mathrm{K}_{1}$ ); el módulo de cortante será igual a:

$$
G=\frac{K_{G}}{K_{1}}
$$

donde:

$\mathrm{K}_{\mathrm{G}}=1,2$, en general
Una vez hechos todos los ensayos anteriores se continua con los ensayos de resistencia en flexión estática. Las condiciones del dispositivo se ilustran en la figura 3, similar al del módulo en flexión. La velocidad de carga se limita y por parte de los laboratorios se utilizan una serie de precauciones que, en el caso de probetas pequeñas, no tendrían ningún sentido, como son las redes de protección contra astillas, ya que éstas pueden saltar con fuerza y causar daño. Comúnmente se colocan micrómetros hasta que se prevé el momento de la fractura, entonces se retiran y se registra sólo la carrera de la prensa. Estos aspectos prácticos no se registran en la norma.

Posteriormente se comprueba, en las cercanías de la zona de fractura, la densidad y el contenido de humedad. Así mismo se anotan en el informe de ensayo todos los condicionantes que se sospechen puedan relacionarse con los resultados. Así, es posible ir refinando o comprobando la veracidad de las condiciones de clasificación, la influencia de factores no reconocidos hasta el momento, las dificultades de realización de los ensayos, etc. Es una novedad de interés al menos en teoría, pero por su carácter poco definido, depende de la voluntad o de la intuición de los técnicos.

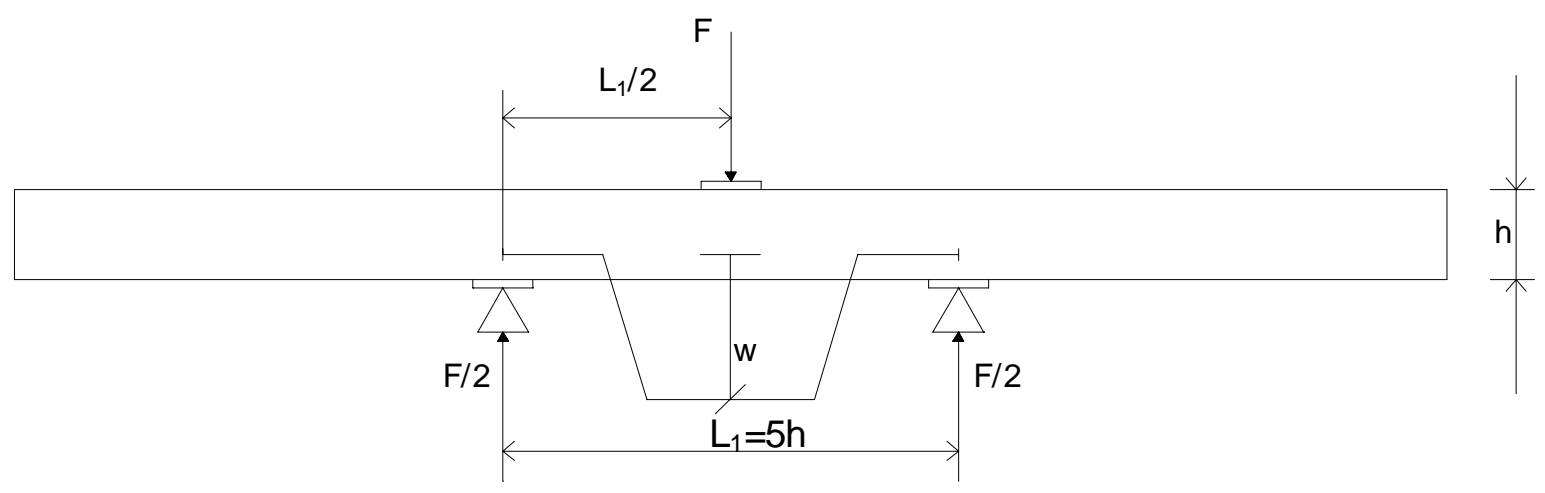

Fig. 4. Dispositivo de ensayo de elasticidad aparente, según UNE-EN 408. 


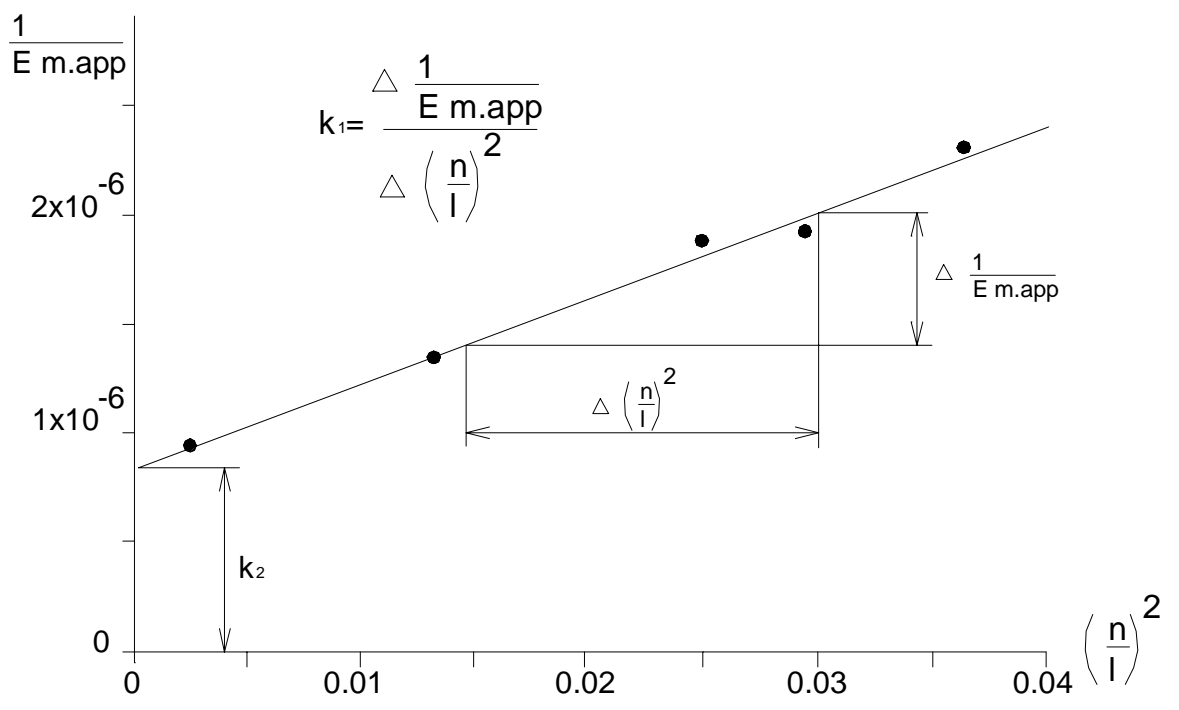

Figura 5. Determinación del módulo de cortante por el método del claro variable. Tomado de UNE-EN 1193.

En el caso de otros ensayos mecánicos ha habido también cambios importantes. Los esfuerzos tangenciales se ensayan con la ayuda de montajes con placas de acero unidas con resinas epoxi o epoxídicas (Fig. 6, ensayo a cortante). Tanto en estos ensayos, como en el caso de ensayar esfuerzos de tracción o compresión en el sentido de la fibra en madera aserrada, se usan del mismo ancho y espesor propios de cada muestra y de una longitud, estimada en función de la dimensión menor. Los dispositivos de ensayo son sencillos.

Un aspecto práctico pero muy relevante es el del secado, más importante aún al tratarse de probetas de determinado tamaño. En los laboratorios del INIA se solucionaron gracias a la experiencia acumulada a lo largo de años de investigación en ese tema, además de que se disponía de unas cámaras de secado rápidas y con resultados óptimos en cuanto a profundidad y homogeneidad del secado. Otros métodos pasan por un secado lento y prohibitivo o bien secados defectuosos que producen deformaciones en las piezas durante el transcurso de los ensayos.

Caben otros estudios paralelos, pues el esfuerzo realizado compensa para correlacionar los resultados, con otros ensayos no registrados en la norma. En el caso de los laboratorios del INIA, se ensayaba además la pieza a flexión, calculando el módulo, en el sentido de menor inercia de la pieza, con una distancia entre apoyos de $900 \mathrm{~mm}$ y utilizando los mismos parámetros físicos de deformación o fuerza límite por cada sección que se usa en la clasificación mecánica, para relacionarlo con el valor obtenido. 


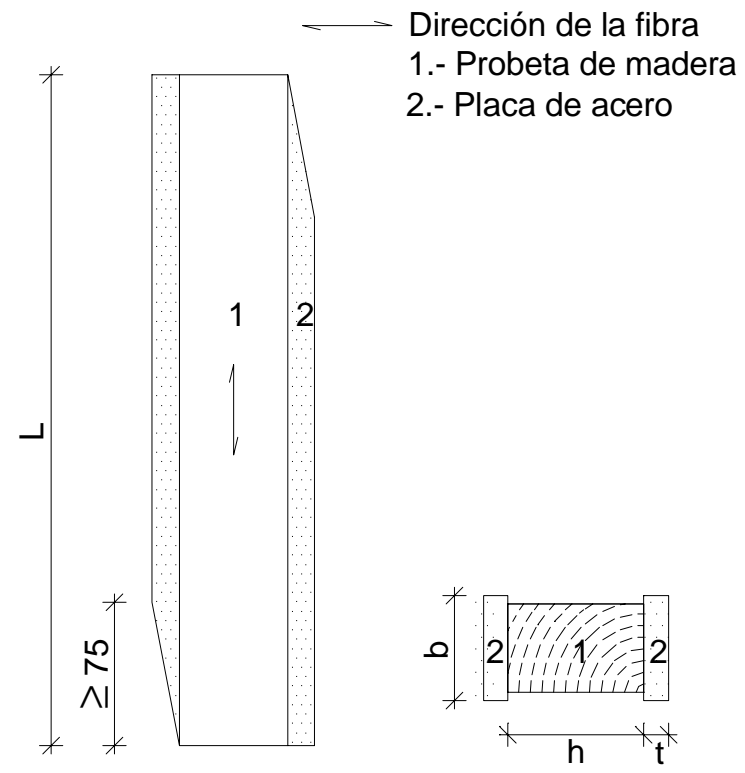

Figura 6. Dispositivos de ensayo a cortante con placas de acero encoladas. Tomado de UNE-EN 1193.

\section{CONCLUSIONES}

Según se pudo apreciar, las novedades se dan tanto en los dispositivos de ensayo, como en la secuencia de ensayos sobre una misma probeta, módulo de cortante y de flexión que culminan en la rotura a flexión. Se trata de una serie mucho más compleja y global que los anteriores métodos de ensayo, algunos de los cuales ni siquiera incluían la determinación del módulo de flexión.

Se puede ver también que el tamaño de muestra descarta producciones pequeñas que, si bien no suelen ser el caso en América, sí son frecuentes en otras zonas. Estos tamaños y la elevada cantidad de ensayos que se han de realizar obligan a disponer de un laboratorio amplio, con sistemas de seguridad y transporte y con gran cantidad de efectivos humanos, lo que encarece enormemente el trabajo, además del costo de la propia muestra, que no es despreciable.
La complejidad de esta normativa, en lo que se refiere a los ensayos, producirá seguramente la reticencia de muchos especialistas, dada esa gran incertidumbre que se tiene con este material. Quizá puede parecer negativo ese exceso de refinamiento teórico al definir distintos módulos y diversidad de propiedades mecánicas y físicas para cada clase resistente, pero debido al planteamiento más complejo y global de los ensayos, en que se emplean gran cantidad de piezas con el tamaño natural de su uso, con defectos y distintas calidades, se ha de convenir que se obtendrán valores numéricos mucho más confiables y reales que los obtenidos con otros métodos con probetas de pequeña dimensión.

Además abre un precedente para ensayar piezas apeadas y en general vigas, aunque no se apliquen los criterios estadísticos. Y, por último, será necesario obtener los valores resistentes de productos provenientes de los Estados Unidos para facilitar su entrada en el mercado europeo. 


\section{BIBLIOGRAFÍA}

AENOR (Asociación Española de Normalización). 1995. Norma UNE-EN 33895. Madera estructural. Clases resistentes. Ed. AENOR. Madrid, España. p:7-8.

AENOR (Asociación Española de Normalización). 1995. UNE-EN 384-95. Madera estructural. Determinación de los valores característicos de las propiedades mecánicas y la densidad. Ed. AENOR. Madrid, España. p:10-13.

AENOR (Asociación Española de Normalización). 1996. UNE-EN 408-96. Estructuras de madera. Madera maciza y madera laminada encolada. Determinación de algunas propiedades físicas y mecánicas. Ed. AENOR. Madrid, España. p:10-20.

AENOR (Asociación Española de Normalización). 1997. UNE-EN 1193-97. Estructuras de madera. Madera estructural y madera laminada encolada. Determinación de la resistencia al esfuerzo cortante y las propiedades mecánicas en dirección perpendicular a la fibra. Ed. AENOR. Madrid, España. p:10-15.

AA.VV. 1998. Certificación Forestal en los Países Nórdicos. AITIM 196:197-206.

AA.VV.; Ministerio de Fomento. 1999. Borrador de norma básica española: estructuras de Madera EM-99. Ed. Ministerio de Fomento. Madrid. Anexo 3.

Argüelles, J. y F. Arriaga. 1992. Curso de diseño y cálculo de estructuras de madera. 1a ed. Ed. Servicio de Publicaciones del Colegio Oficial de Arquitectos de Madrid. Madrid. p:23-26.
Fernández-Golfín, J.I.; R. Díez y A. Gutiérrez. 1999. Coníferas españolas de uso estructural. Rev. AITIM № 200. p: $72-75$.

IRANOR (Instituto Nacional de Racionalización y Normalización). 1978. UNE 56-528. Características físicomecánicas de la madera. Preparación de probetas para ensayo.

IRANOR (Instituto Nacional de Racionalización y Normalización). 1978. UNE 56-529. Características físicomecánicas de la madera. Determinación del contenido de humedad por desecación hasta el estado anhidro. Ed. IRANOR. Madrid, España. p:1.

IRANOR (Instituto Nacional de Racionalización y Normalización). 1978. UNE 56.531. Características físicomecánicas de la madera. Determinación del peso específico. Ed. IRANOR. Madrid, España.

IRANOR (Instituto Nacional de Racionalización y Normalización). 1978. UNE 56. 535. Características físicomecánicas de la madera. Determinación de la resistencia a la compresión axial. Ed. IRANOR. Madrid, España.

IRANOR (Instituto Nacional de Racionalización y Normalización). 1978. UNE 56-536. Características físicomecánicas de la madera. Determinación de la resistencia a la flexión dinámica. Ed. IRANOR. Madrid, España.

IRANOR (Instituto Nacional de Racionalización y Normalización). 1978. UNE 56-537. Características físicomecánicas de la madera. Determinación de la resistencia a la flexión estática. Ed. IRANOR. Madrid, España. 
IRANOR (Instituto Nacional de Racionalización y Normalización). 1978. UNE 56-538. Características físicomecánicas de la madera. Determinación de la resistencia a la tracción perpendicular a las fibras. Ed. IRANOR. Madrid, España.

IRANOR (Instituto Nacional de Racionalización y Normalización). 1978. UNE 56-539. Características físicomecánicas de la madera. Determinación de la resistencia a la hienda. Ed. IRANOR. Madrid, España.

IRANOR (Instituto Nacional de Racionalización y Normalización). 1978. UNE 56-540. Características físicomecánicas de la madera. Interpretación de los resultados de ensayo. Ed. IRANOR. Madrid, España.

J. Perís, A. Vignote. 1999. Tecnología de la madera. $3^{\mathrm{a}}$ ed. Ed. Mundiprensa. Madrid. p:203-215.
Orús, F. 1985. Materiales de construcción. $2^{\mathrm{a}}$ ed. Ed. Dossat. Madrid. p:505510 .

Peraza O., C. 1990. Estructuras de madera. $1^{\text {a }}$ ed. Ed. Fundación Escuela de la Edificación. Madrid. p: III 21-28.

\section{Glosario de términos más empleados en México}

AA.VV. Autores varios.

Fendas. Agrietamientos o fisuras de la madera en sentido radial, en general debido a problemas de secado.

Flexotracción. Flexotensión.

Hormigón. Concreto.

Minoraciones. Factores de ajuste.

Tensiones básicas. Esfuerzos básicos

Tracción. Tensión. $\diamond$

1 Departamento Construcciones Arquitectónicas I. E.T.S. Arquitectura. Universidad de Sevilla España. Av. Reina Mercedes Núm. 2. Sevilla 41006 España.

Manuscrito recibido el 15 de agosto de 2001.

Aceptado el 16 de noviembre de 2001.

Este documento se debe citar como:

Ariza L., I. 2002. El nuevo enfoque en los ensayos mecánicos de la madera aserrada para uso estructural en la normativa europea. Madera y Bosques 8(2):3-16. 